\title{
Desempenho de metal de solda adequado para soldagem de componentes de ancoragem
}

\section{Performance of high strength steel weld metal for welding of mooring components}

\author{
Antonio José de Carvalho Gomes ${ }^{1}$, Jorge Carlos Ferreira Jorge ${ }^{1}$, \\ Luís Felipe Guimarães de Souza ${ }^{1}$, Ivani de Souza Bott ${ }^{2}$, \\ Leonardo Sales Araújo ${ }^{3}$, Matheus Campolina Mendes ${ }^{1}$
}

\footnotetext{
${ }^{1}$ Centro Federal de Educação Tecnológica Celso Suckow da Fonseca - CEFET/RJ, Diretoria de Pesquisa e Pós- graduação - DIPPG, Rio de Janeiro, RJ, Brasil.

${ }^{2}$ Pontificia Universidade Católica, PUC-RIO, Departamento de Engenharia Química e de Materiais - DEQM, Rio de Janeiro, RJ, Brasil.

${ }^{3}$ Universidade Federal do Rio de Janeiro - UFRJ, Instituto Alberto Luiz Coimbra de Pós-graduação e Pesquisa de Engenharia - COPPE, Rio de Janeiro, RJ, Brasil.

e-mail: antoni.cg@hotmail.com, ivanibott@gmail.com, jorgecfjorge@gmail.com, lfgs59@gmail.com, campolinamendes@gmail.com, leonardo.sales@gmail.com
}

\section{RESUMO}

O presente trabalho apresenta os resultados de um programa de pesquisa cujo objetivo principal é o desenvolvimento de consumíveis adequados para a soldagem de componentes de ancoragem fabricados em aços de alta resistência. Após avaliação de diferentes composições químicas, com base no balanço de Mn e Ni é feita uma análise detalhada das propriedades mecânicas e microestruturais da composição química que propiciou os melhores resultados para permitir uma conclusão decisiva.

Foram soldadas juntas pelo processo eletrodo revestido utilizando consumíveis de 4,0 mm de diâmetro, com preaquecimento de $200{ }^{\circ} \mathrm{C}$, corrente contínua, posição plana e energia de soldagem nominal de $1,5 \mathrm{~kJ} / \mathrm{mm}$. Após a soldagem, foram realizados ensaios mecânicos (tensão, impacto Charpy-V, dureza) e metalográficos por microscopia ótica, microscopia eletrônica de varredura e EBSD em amostras removidas integralmente do metal de solda, tanto na condição de como soldado quanto após tratamento térmico pós-soldagem (TTPS) realizado a $600{ }^{\circ} \mathrm{C}$. Os resultados mostram que os metais de solda obtiveram propriedades mecânicas satisfatórias após o TTPS, sendo adequados para aplicação em componentes de ancoragem de acordo com a especificação IACS W22. Além disso, observou-se que a tenacidade ao impacto experimentou uma melhoria com o aumento do tempo de TTPS, o que é crucial para componentes com maiores espessuras.

Palavras-chave: metal de solda, propriedades mecânicas, tratamento térmico, componentes de ancoragem.

\section{ABSTRACT}

The present work show the results of an extensive working program whose the main goal is the development of consumables suitable for welding of mooring components manufactured in high strength steels. After an exhaustive evaluation of different chemical compositions, based on a balance of $\mathrm{Mn}$ and $\mathrm{Ni}$, a detailed analysis of the mechanical and microstructural properties is done for the heat which proposed the best results in order to allow a decisive conclusion.

Welded joints were produced by using $4,0 \mathrm{~mm}$ diameter covered electrodes, with preheat of $200{ }^{\circ} \mathrm{C}$, direct current, flat position and nominal heat input of $1.5 \mathrm{~kJ} / \mathrm{mm}$. After welding, mechanical tests (tension, impact Charpy-V, hardness) and metallographic examination (optical microscopy and scanning electron microscopy, electron back scattering diffraction) were performed in specimens removed integrally from the weld metal both in the as welded condition and post weld heat treatment at $600{ }^{\circ} \mathrm{C}$. The results show that the weld metals obtained satisfactory mechanical properties after PWHT, being adequate for application on high strength steel mooring components with several grades according to the IACS W22 specification. In addition, 
it was noted that impact Charpy-V results were improved with increasing the time of PWHT, which is crucial for larger components.

Key words: weld metal, mechanical properties, heat treatment, mooring components.

\section{1. INTRODUÇÃO}

A crescente demanda mundial por petróleo e gás tem motivado a busca de fontes de energia em águas profundas. As plataformas ancoradas oferecem uma solução atraente para prospecção de petróleo e gás em variadas profundidades de lâmina d'água no leito marinho [1]. Uma unidade flutuante offshore está sujeita à ação de diversas forças ambientais e as cargas produzidas pela combinação do vento, ondas e correntes levam a um carregamento alternado e complexo, favorecendo a ocorrência de falha por fadiga e propagação de trincas [2].

O principal objetivo de um sistema de ancoragem é restringir o movimento decorrente das forças ambientais na extração de óleo e, consequentemente, permitir a operação segura do equipamento durante a produção [3]. As linhas de ancoragem para águas profundas consistem em longos comprimentos de elos de corrente de aço, cabos de aço ou cabos de poliéster e outros acessórios [4,5]. Normalmente, essas linhas são projetadas para uma vida operacional de cerca de 20 anos e inspeções periódicas são obrigatórias para monitorar a integridade estrutural desses componentes. A falha de um único elemento em uma linha de ancoragem pode promover graves danos ambientais e econômicos.

À medida que os campos de exploração dos recursos de petróleo e gás se expandem para maiores profundidades, aumenta-se a necessidade de redução de peso das estruturas e materiais com maior resistência e tenacidade são necessários [6]. Com base neste cenário, as normas para seleção de materiais e aplicação em componentes de ancoragem apresentam um conjunto de requisitos muito rigorosos, como mostrado na Tabela 1. Estes requisitos estão de acordo com a International Association Classification Society (IACS) [7]. Para alcançar estes requisitos, alguns aços de alta resistência são reconhecidos por terem aplicações importantes na fabricação de componentes para unidades com sistemas de ancoragem offshore [3,8-15]. A Tabela 2 mostra algumas composições químicas específicas utilizadas para esta aplicação, onde é de destaque, além da presença de elementos de liga cruciais para a temperabilidade, o limitado teor de carbono com o objetivo de melhorar a tenacidade e soldabilidade.

Tabela 1: Propriedades mecânicas exigidos para amarras e acessórios de ancoragem [7].

\begin{tabular}{c|c|c|c|c|c|c}
\hline Grau & $\begin{array}{c}\text { LE } \\
(\mathbf{M P a})\end{array}$ & $\begin{array}{c}\text { LR } \\
(\mathbf{M P a})\end{array}$ & $\begin{array}{c}\text { Al } \\
(\boldsymbol{\%})\end{array}$ & $\begin{array}{c}\text { RA } \\
(\boldsymbol{\%})\end{array}$ & $\begin{array}{c}\text { Energia de impacto } \\
\text { Charpy-V à -20 } \mathbf{C}(\mathbf{J})\end{array}$ & $\begin{array}{c}\text { Energia de impacto Charpy- } \\
\mathbf{V}_{\mathbf{~ a ̀ ~}}^{-20} \mathbf{C}(\mathbf{J})(\mathbf{s o l d a})\end{array}$ \\
\hline R3 & 410 & 690 & 17 & 50 & 40 & 30 \\
\hline R3S & 490 & 770 & 15 & 50 & 45 & 33 \\
\hline R4 & 580 & 860 & 12 & 50 & 50 & 40 \\
\hline R4S & 700 & 960 & 12 & 50 & 56 & 42 \\
\hline R5 & 760 & 1000 & 12 & 50 & 58 & 36 \\
\hline
\end{tabular}

Onde: LE - Limite de escoamento, LR - Limite de resistência, Al - Alongamento, RA-Redução de área.

Tabela 2: Composição química de materiais utilizados em componentes de ancoragem [2,8-15].

\begin{tabular}{c|c|c|c|c|c|c|c|c|c}
\hline Ref & Componente & $\begin{array}{c}\text { Processo de } \\
\text { fabricação }\end{array}$ & $\begin{array}{c}\text { LR } \\
(\mathbf{M P a})\end{array}$ & $\mathbf{C}$ & $\mathbf{M n}$ & $\mathbf{C r}$ & $\mathbf{N i}$ & Mo & Ceq(*) \\
\hline 2 & Elo de amarra & $\mathrm{L}$ & 877 & 0.24 & 1.06 & 1.05 & 0.55 & 0.22 & 0.71 \\
\hline 5 & Elo de amarra & $\mathrm{L}$ & 1201 & 0.23 & 1.48 & 0.65 & 0.76 & 0.56 & 0.77 \\
\hline 6 & Elo de amarra & $\mathrm{L}$ & 699 & 0.32 & 1.69 & 0.10 & 0.05 & 0.01 & 0.63 \\
\hline 7 & Elo de amarra & $\mathrm{L}$ & 716 & 0.31 & 1.60 & 0.04 & 0.01 & 0.07 & 0.59 \\
\hline 8 & Manilha & $\mathrm{Fd}$ & 704 & 0.24 & 1.37 & 0.45 & 0.69 & 0.32 & 0.63 \\
\hline 9 & Manilha & $\mathrm{Fd}$ & 880 & 0.21 & 0.80 & 0.55 & 2.78 & 0.31 & 0.70 \\
\hline 4 & Olhal & $\mathrm{Fd}$ & 766 & 0.25 & 1.17 & 0.58 & 0.59 & 0.35 & 0.67 \\
\hline 10 & Conector & $\mathrm{Fj}$ & 730 & 0.27 & 0.53 & 0.93 & 0.19 & 0.21 & 0.60 \\
\hline 11 & Conector & $\mathrm{Fj}$ & 754 & 0.31 & 0.92 & 0.97 & 0.88 & 0.42 & 0.81 \\
\hline
\end{tabular}

Onde: Fd- Fundido; Fj - Forjado; L - Laminado; (*)Ceq = C + Mn/6 + (Cr+Mo+V)/5 + (Ni+Cu)/15.

De fato, apesar de existirem acessórios como manilhas e soquetes de ancoragem (Figura 1) fabricados por outros processos tais como forjamento ou fundição, a execução de operações de soldagem é usual para 
reparo de defeitos de fabricação, com o objetivo de evitar o sucateamento prematuro do componente. Nestes casos, realiza-se de forma obrigatória um tratamento térmico pós-soldagem [16-18] após o reparo, com o objetivo de ajustar as propriedades mecânicas e aliviar as tensões residuais [10].

O cenário discutido acima torna o desenvolvimento de consumíveis de soldagem apropriado para esta aplicação um desafio complexo, uma vez que não há normas disponíveis envolvendo este elevado nível de resistência. De fato, a norma AWS geralmente utilizada para qualificação de consumíveis de soldagem [20] é limitada a $120 \mathrm{ksi}(830 \mathrm{MPa})$ e não prevê requisitos após o tratamento térmico pós-soldagem (TTPS). Assim, para alcançar as propriedades mecânicas mostradas na Tabela 1, os consumíveis especiais são avaliados caso a caso [21]. Neste aspecto, trabalhos prévios [21-23] demonstraram que a presença dos elementos $\mathrm{Cr}$ e $\mathrm{Mo}$, embora tendo uma contribuição significativa para o aumento da resistência mecânica, pode promover efeitos nocivos na tenacidade ao impacto após TTPS. Isso faz com que a obtenção de resultados satisfatórios seja uma tarefa muito complexa. Embora existam consumíveis comerciais que já tenham propiciado um bom desempenho [21-28], é importante destacar que por vezes estes podem não estar disponíveis no mercado nacional fazendo com que importantes projetos de engenharia sejam interrompidos. De modo a superar este problema, foi implementado um extenso programa de pesquisa para desenvolver um consumível nacional adequado para esta aplicação utilizando diferentes composições químicas e procedimentos de soldagem [13,2124,29-32].
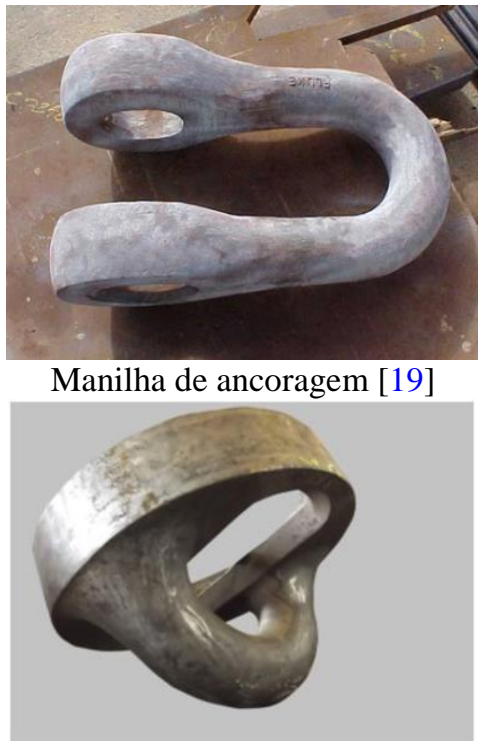

Olhal de torpedo [8]
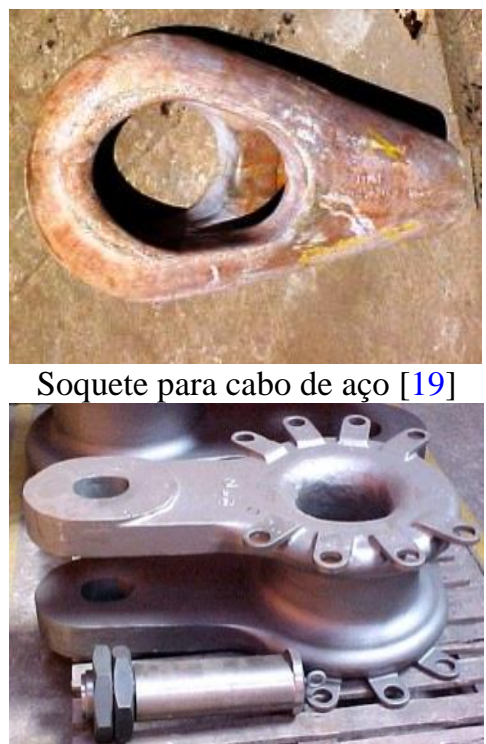

Manilha para cabo de poliester [5]

Figure 1: Exemplos de acessórios usados em linhas de ancoragem [5,8,19].

O presente trabalho avalia as propriedades mecânicas e microestruturais de metal de solda contendo teores balanceados de $\mathrm{Ni}$ e $\mathrm{Mn}$ e baixo $\mathrm{Cr}$ que apresentou o melhor desempenho no programa de pesquisa e realiza uma análise de sua adequação para utilização na soldagem de componentes de ancoragem.

\section{MATERIAIS E MÉTODOS}

\subsection{Materiais}

O consumível de soldagem empregado foi um eletrodo revestido com 4,0mm de diâmetro. A composição química do metal de solda obtida por espectroscopia de emissão ótica na linha central do cordão de solda é mostrado na Tabela 3.

Chapas de aço carbono ASTM A 36 com dimensões de 19 x 300x 700 mm (espessura x largura x comprimento) foram usadas como material base. 
Tabela 3: Composição química do metal de solda depositado (\% peso).

\begin{tabular}{c|c|c|c|c|c|c|c|c|c|c}
\hline Elemento & $\mathbf{C}$ & $\mathbf{S i}$ & $\mathbf{P}$ & $\mathbf{S}$ & $\mathbf{M n}$ & $\mathbf{M o}$ & $\mathbf{N i}$ & $\mathbf{C r}$ & $\mathbf{T i}$ & $\mathbf{V}$ \\
\hline Peso, \% & 0,05 & 0,14 & 0,02 & 0,01 & 1,91 & 0,39 & 2,97 & 0,26 & 0,007 & 0,005 \\
\hline
\end{tabular}

\subsection{Soldagem}

A Figura 2 mostra a geometria da junta utilizada no presente trabalho, a qual foi modificada de acordo com a especificação ISO 15792 [33] de modo a eliminar o efeito de diluição com o metal base.

A soldagem foi realizada na posição plana pelo processo eletrodo revestido com preaquecimento de 200 ${ }^{\circ} \mathrm{C}$, utilizando os parâmetros apresentados na Tabela 4.

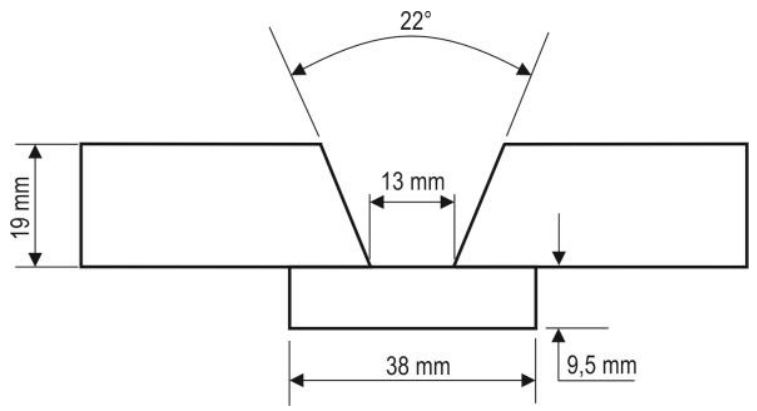

Figura 2: Geometria da junta soldada (mm).

Tabela 4: Parâmetros de soldagem utilizados.

\begin{tabular}{c|c|c|c}
\hline Corrente (A) & Tensão (Volts) & Energia de soldagem (kJ/mm) & Número de passes \\
\hline $185-195$ & $22-30$ & 1,51 & 18 \\
\hline
\end{tabular}

\subsection{Tratamento térmico pós-soldagem (TTPS)}

Os metais de solda foram testados nas condições de como soldado e tratados termicamente. O tratamento térmico pós-soldagem (TTPS) foi realizado a $600{ }^{\circ} \mathrm{C}$ por 1,2 e 3 horas, seguido de resfriamento ao ar.

A temperatura de $600{ }^{\circ} \mathrm{C}$ foi usada para o TTPS, porque essa temperatura é a geralmente recomendada para alívio das tensões residuais de equipamentos de ancoragem [21-23].

\subsection{Ensaios mecânicos}

Foram realizados ensaios de tração à temperatura ambiente conforme as normas ASTM A 370 [34] e ASTM E8 [35], em corpos de prova cilíndricos de diâmetro $8,75 \mathrm{~mm}$ e comprimento de $50 \mathrm{~mm}$, removidos de forma longitudinal e integralmente dos cordões de solda.

Foram realizados ensaios de impacto Charpy-V em temperaturas variando de -60 a $20{ }^{\circ} \mathrm{C}$ em corpos de prova padronizados (10x10x55mm) de acordo com as normas ASTM A 370 [34] e ASTM E23 [36], removidos da linha central dos cordões à $2 \mathrm{~mm}$ da superfície da junta soldada (Figura 3 ). As curvas de transição foram obtidas usando 3 corpos de prova para cada condição.

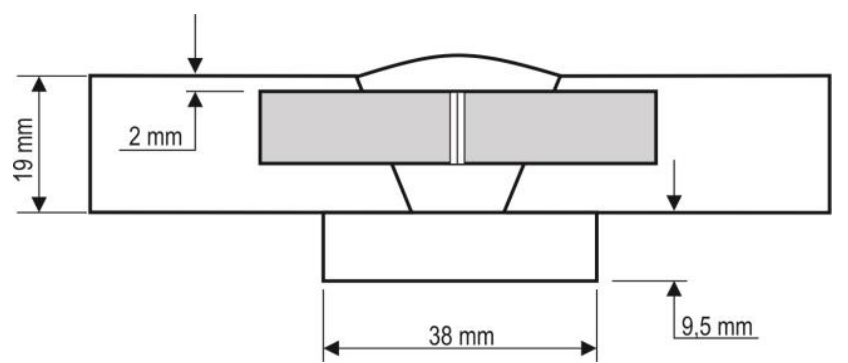

Figura 3: Desenho esquemático da posição dos corpos de prova para ensaio de impacto Charpy-V. 
Foram realizados ensaios de microdureza Vickers $\left(\mathrm{HV}_{0,5}\right)$ com aplicação de carga de 500 gf. em corpos de prova removidos dos metais de solda. As medições foram feitas em três linhas paralelas separadas por $0,25 \mathrm{~mm}$ da superfície superior até a raiz com espaçamento de $1,0 \mathrm{~mm}$, como mostra a Figura 4.

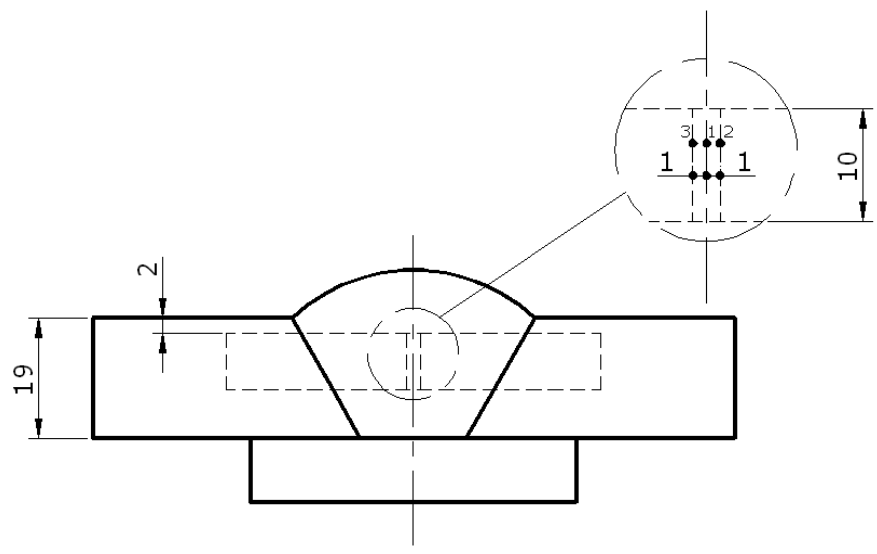

Figure 4: Posição das medições de microdureza Vickers $\left(\mathrm{HV}_{0.5}\right)$.

\subsection{Ensaios Metalográficos}

Foi realizada a caracterização microestrutural dos metais de solda por microscopia ótica (MO) e eletrônica de varredura (MEV) na posição relativa ao entalhe do corpo de prova de impacto Charpy-V.

Foi ainda realizada análise por Electron Back Scatering Diffraction (EBSD) para observação de detalhes adicionais, tais como frequência de contornos de alto ângulo $\left(>15^{\circ}\right)$ [37], grau de recristalização com menor energia de deformação [38] e tamanho de grão efetivo (TGE). Os mapas de EBSD foram coletados com o MEV operando a $20 \mathrm{kV}$ e step-size de $0,3 \mu \mathrm{m}$. Um pós-processamento dos dados de EBSD foi realizado usando o pacote de software HKL Channel 5.

Os componentes da matriz foram classificados em grãos recristalizados, subestruturados e deformados de acordo com a desorientação média entre os grãos (GAM) para distinguir a diferença de energia de deformação local. Os grãos recristalizados têm a menor energia de deformação local, enquanto os grãos deformados têm a maior energia de deformação local [38].

O preparo das amostras consistiu do procedimento convencional de lixamento e polimento, seguido de ataque químico com nital $2 \%$.

Para análise por EBSD, foi realizado um polimento adicional com sílica coloidal 0,06 $\mu \mathrm{m}$ e as amostras foram observadas sem ataque químico.

\section{RESULTADOS}

\subsection{Ensaios Mecânicos}

A Tabela 5 mostra os resultados dos ensaios de tração, onde se observam as seguintes características principais:

a) Os resultados superiores foram obtidos na condição de como soldado;

b) O TTPS promoveu uma redução na resistência mecânica para todas as condições e;

c) $\mathrm{O}$ aumento do tempo de tratamento provoca uma redução contínua da resistência mecânica.

Tabela 5: Resultados dos ensaios de tração.

\begin{tabular}{c|c|c|c|c}
\hline Condição & LE (MPa) & LR (MPa) & Al (\%) & RA (\%) \\
\hline Como soldado & 822 & 896 & 18 & 58 \\
\hline TTPS-1h & 806 & 888 & 21 & 60 \\
\hline TTPS-2h & 755 & 844 & 20 & 62 \\
\hline TTPS-3h & 723 & 797 & 23 & 64 \\
\hline
\end{tabular}


As Figuras 5 e 6 mostram os resultados dos ensaios de Charpy-V, onde se observam as seguintes características principais:

a) O TTPS promoveu uma melhoria na tenacidade ao impacto;

b) Resultados superiores de tenacidade ao impacto são obtidos com o aumento do tempo de tratamento e;

c) Todos os resultados são aceitáveis para os graus R3, R3S e R4 após o TTPS.

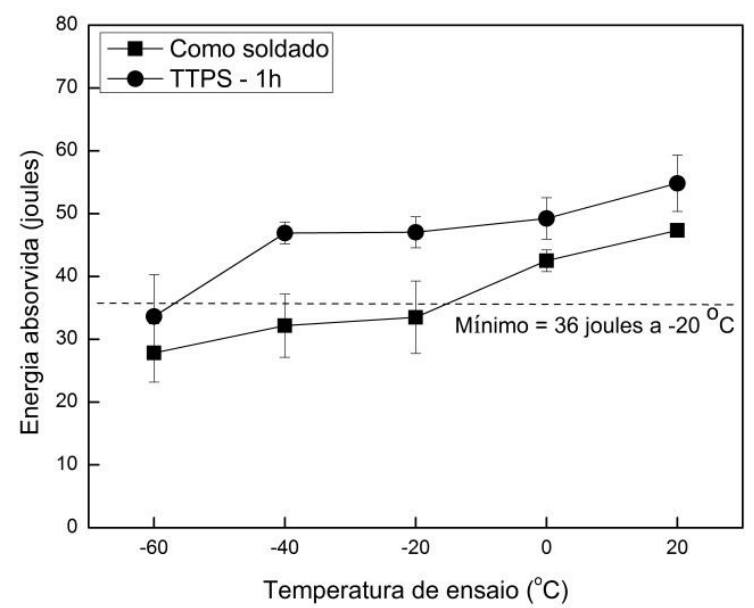

Figure 5: Curva de transição do metal de solda.

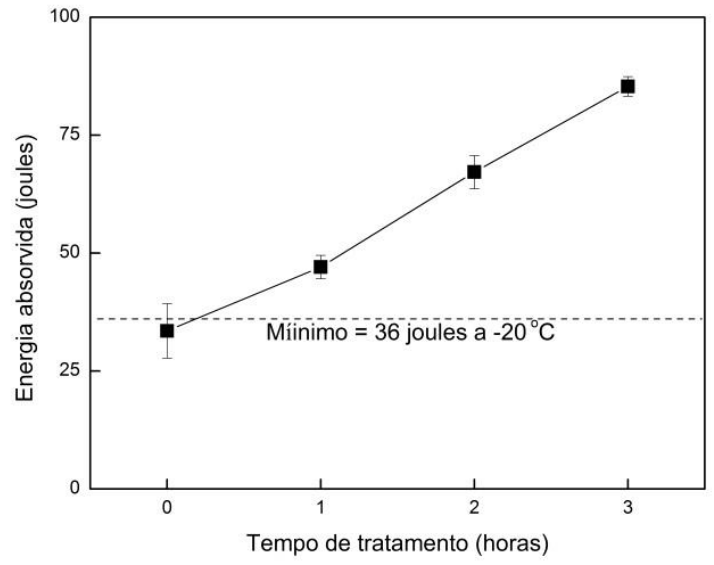

Figura 6: Influência do tempo de tratamento na tenacidade ao impacto a $-20{ }^{\circ} \mathrm{C}$.

A Figura 7 mostra os resultados do ensaio de microdureza onde se nota o efeito do TTPS. O comportamento acompanha a tendência observada para o limite de resistência (Tabela 4). 


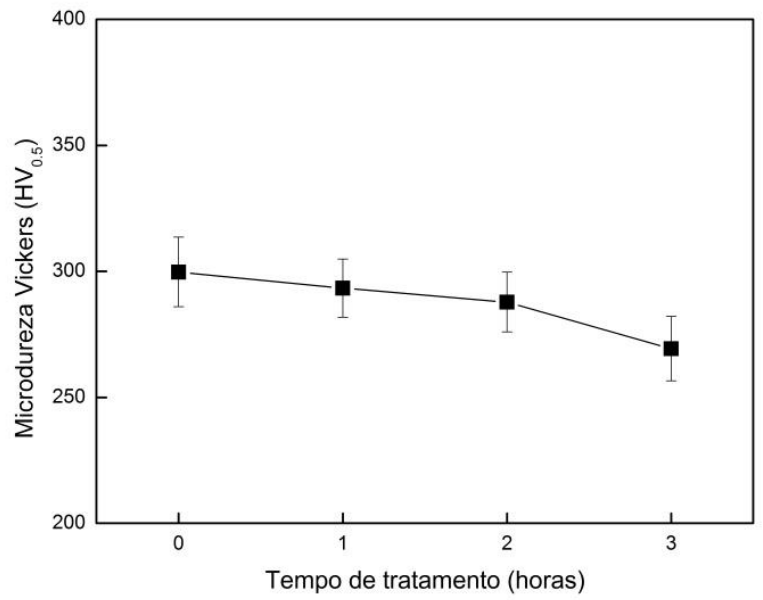

Figura 7: Influência do tempo de tratamento na microdureza Vickers.

\subsection{Ensaios Metalográficos}

A Figura 8 mostra a macrografia da junta soldada, onde se nota o efeito da soldagem multipasses e a ausência de defeitos rejeitáveis.

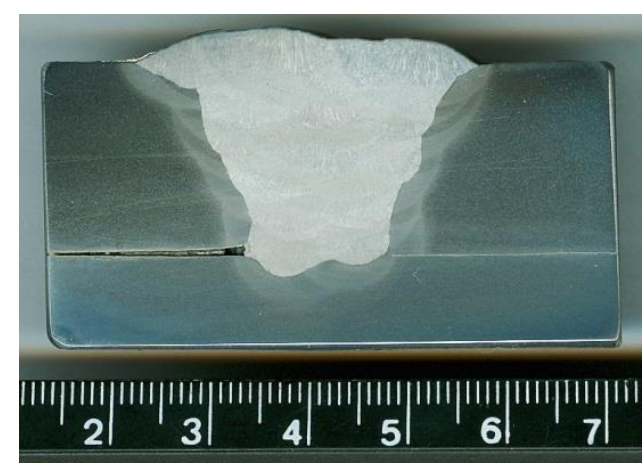

Figura 8: Macrografia da junta soldada. Ataque: nital 2\%.

A Figura 9 mostra as micrografias das regiões do metal de solda, onde se observa a predominância de martenstia na região do último passe e uma mistura de martensita e bainita na posição relativa ao entalhe Charpy-V, embora ainda com predomínio de martensita.

A influência do TTPS nas características do metal de solda é mostrada nas Figuras 10 e 11 . O mapa de deformação (Figura 10) mostra que a maioria dos grãos foram categorizados como grãos deformados de alta energia de deformação na condição de como soldado, enquanto uma mudança significativa é observada após TTPS. Além disso, a maior frequência de contornos de alto ângulo (CAA) é vista após TTPS (Figura 11). Assim, pode-se inferir que o TTPS além do revenimento da microestrutura, contribui para a ocorrência de outras características benéficas para a tenacidade ao impacto. 

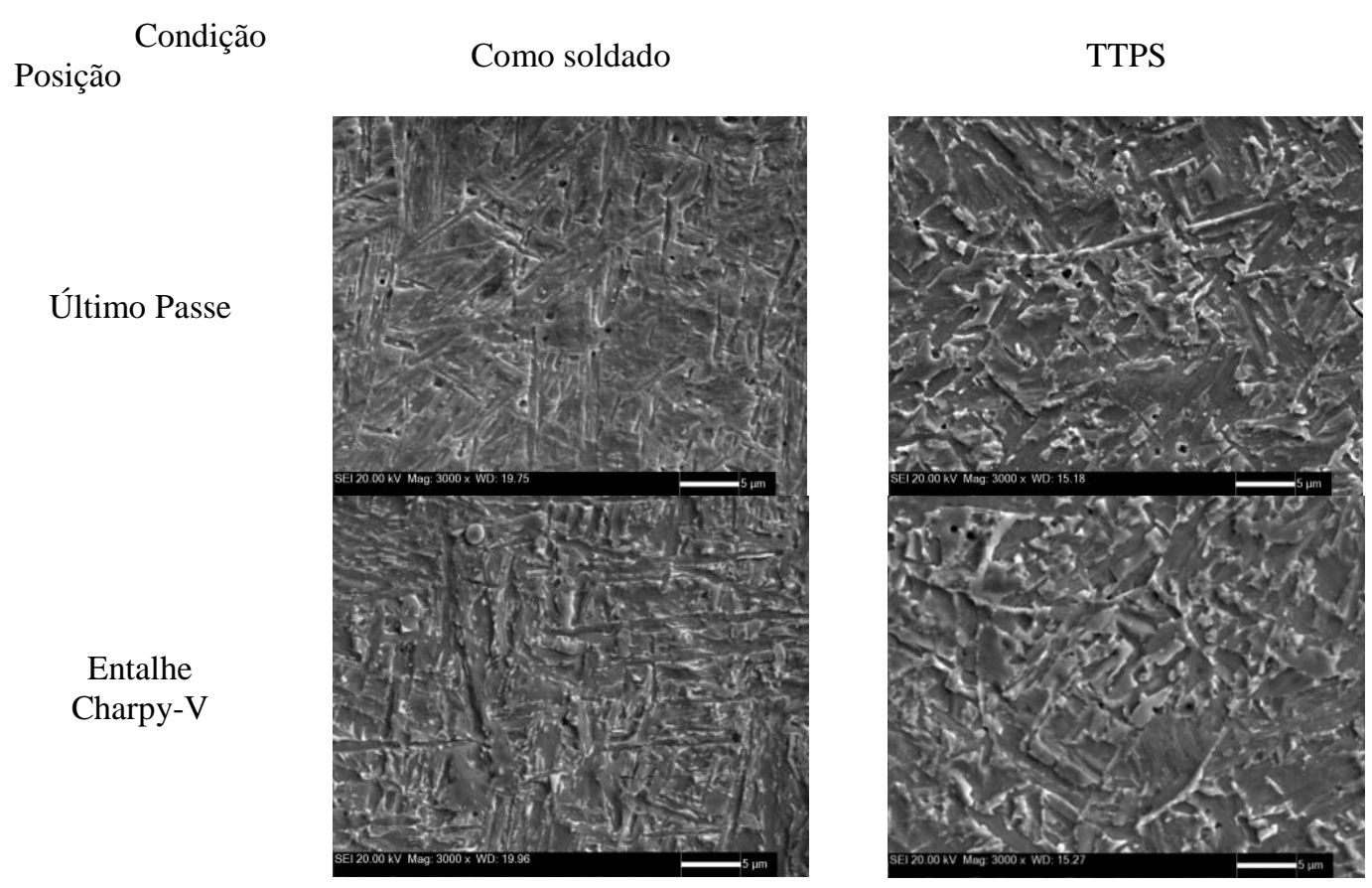

Figure 9: Microestrutura do metal de solda (MEV). Ataque: nital 2\%.

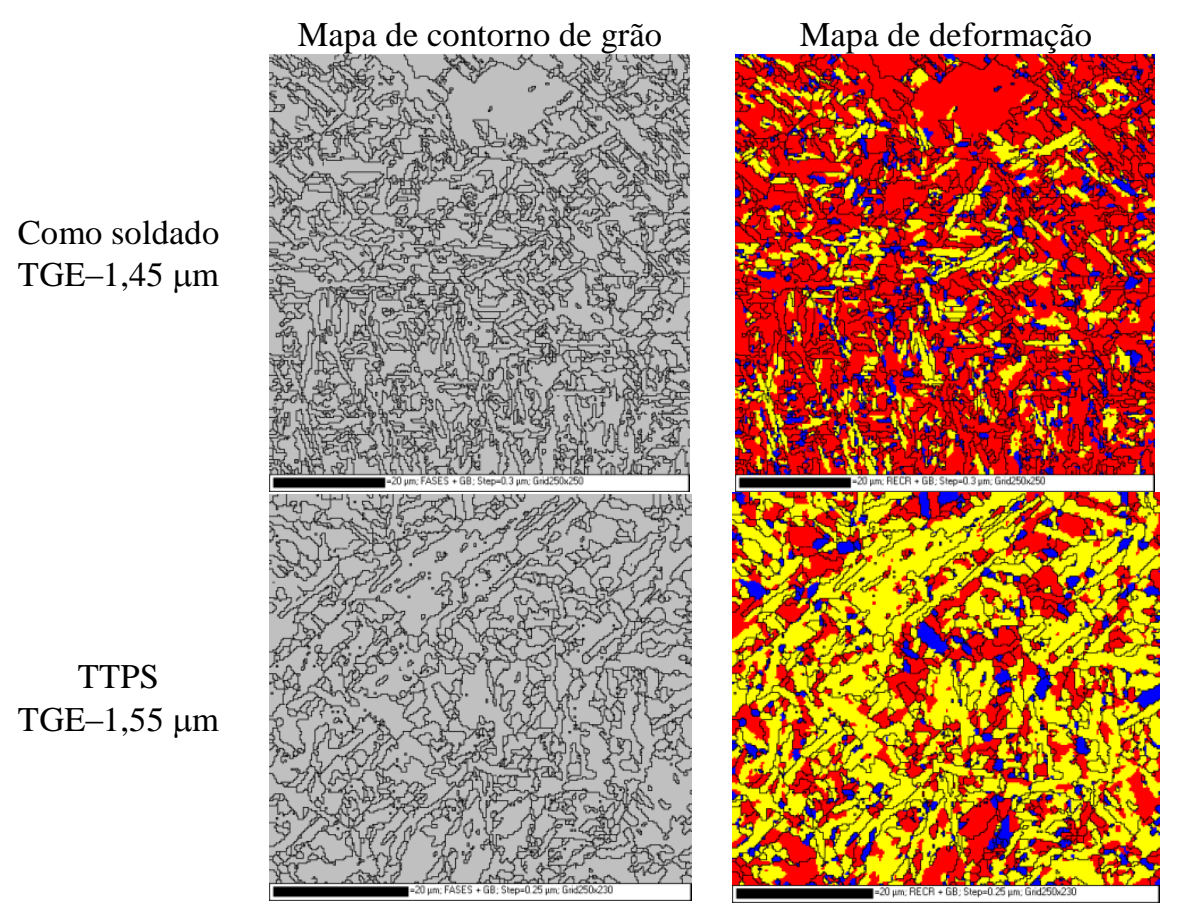

Figure 10: Influência do TTPS nas características do metal de solda (EBSD).

Onde: TGE - Tamanho de Grão Efetivo. Grãos recristalizados (azul), sub-estruturados (amarelo) e deformados (vermelho). 


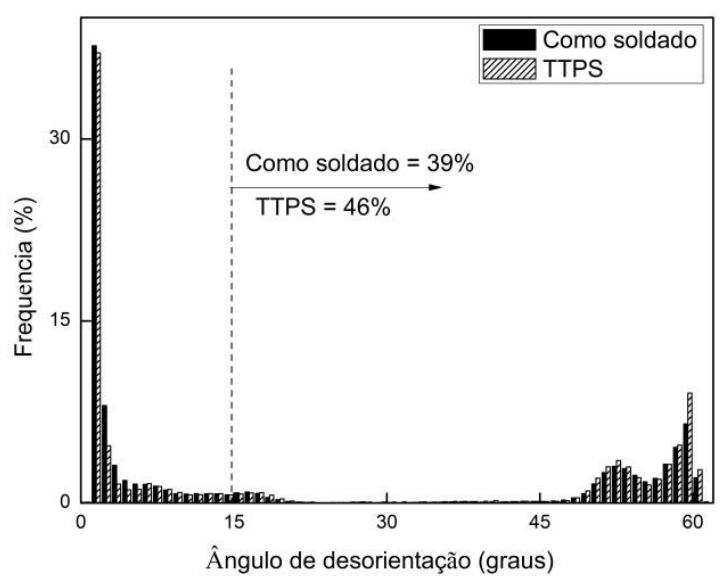

Figura 11: Influência do TTPS na frequência de contornos de alto ângulo do metal de solda.

\section{DISCUSSÃO}

\subsection{Propriedades Mecânicas}

Quando da análise dos requisitos para os componentes de ancoragem (Tabela 1), fica claro o desafio para atingir os níveis requeridos de resistência e tenacidade, simultaneamente. Além da grande dificuldade de obtenção dos valores de resistência mecânica para o metal de solda [39], é esperada uma queda da tenacidade para estes elevados níveis de resistência [40].

De uma forma geral, uma tenacidade ao impacto adequada depende dos teores de Ni e Mn [21,41-47], sendo necessário um balanço adequado destes elementos para manter uma boa relação entre resistência e tenacidade [47]. Particularmente, uma composição com a adição de 2,0Mn-3,0Ni-0,5Cr tem sido indicada para este propósito [22,28]. Embora, o elemento $\mathrm{Cr}$ seja nocivo para a tenacidade, a sua adição é usual para que se obter os níveis de resistência desejados [48].

A Figura 12 mostra uma comparação dos resultados obtidos no presente trabalho com outros trabalhos experimentais [18-22,24-26,49-59], onde se observa que os resultados atuais parecem ser inferiores, uma vez que a tenacidade ao impacto não é tão elevada como as obtidas em outras investigações. Estes resultados são consequência da microestrutura composta por martensita e bainita, mas também com ocorrência de componentes de baixa tenacidade [28,60], tais como a bainita coalescida (Figura 13) em algumas regiões.

Entretanto, deve-se ressaltar que este não é um problema crítico na soldagem de aços de alta resistência usados em componentes de ancoragem, uma vez que o TTPS é sempre aplicado [19,21-24,29-32].

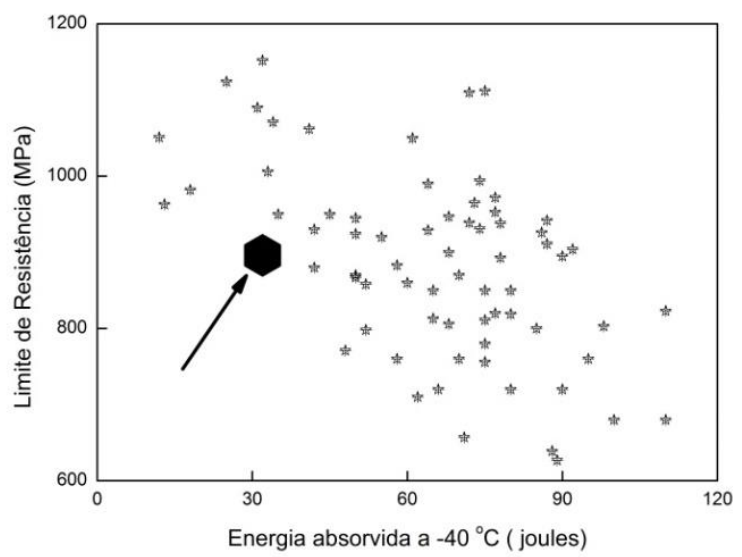

Figura 12: Relação entre a resistência mecânica e energia absorvida $\left(-40{ }^{\circ} \mathrm{C}\right)$ após soldagem obtida no presente trabalho (ponto indicado pela seta) em comparação com outros resultados disponíveis na literatura [18-22,24-26,49-59]. 


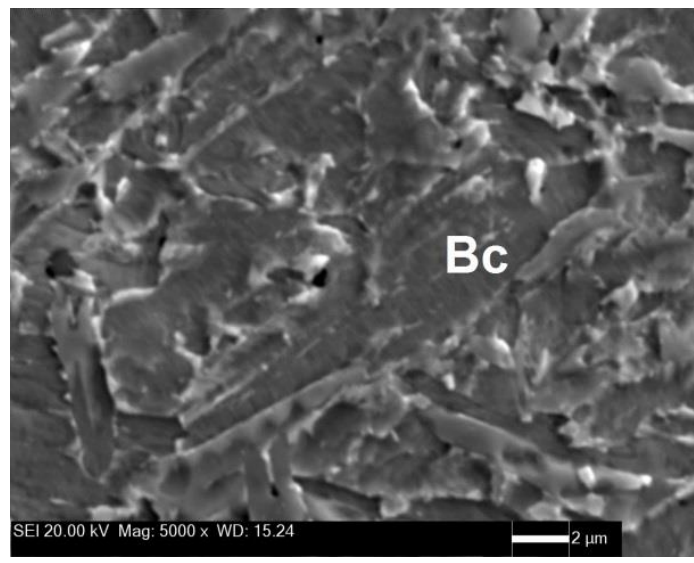

Figura 13: Ocorrência de bainita coalescida (Bc) no metal de solda (MEV). Ataque: nital 2\%.

De fato, para o metal de solda desenvolvido (Tabela 3), a execução do TTPS promoveu um aumento na tenacidade ao impacto como pode ser visto na Figura 6, como consequência das alterações observadas na microestrutura, tais como revenido da martensita, redução da densidade de discordâncias e aumento da frequência dos contornos de alto ângulo. Esta foi a principal razão para estudar uma composição química com base em maiores teores de $\mathrm{Mn}$ e $\mathrm{Ni}$ e baixo teor de $\mathrm{Cr}$, devido ao efeito nocivo do $\mathrm{Cr}$ na tenacidade ao impacto após TTPS [21-23,28,61-66].

As características discutidas acima, propiciaram a obtenção dos requisitos dos aços mais utilizados em componentes de ancoragem no Brasil, aços de grau R3 e grau R4 (Tabela 1). Como visto na Tabela 4 e na Figura 6, a influência do tempo de revenimento é relevante principalmente para os níveis de resistência mecânica e, como já apontado por Surian et al. [39], este é o principal desafio para o desenvolvimento de consumíveis de soldagem de elevada resistência mecânica.

\subsection{Aspectos Práticos}

Os progressos na tecnologia de fabricação de aços têm motivado novos desenvolvimentos também em processos e consumíveis de soldagem para obtenção de metais de solda com propriedades mecânicas essencialmente equivalentes as do metal de base $[67,68]$. Isto é mais crítico para níveis de resistência superiores a 830MPa, onde não existem normas específicas para aprovação de consumíveis de soldagem. Além disso, para aplicações similares as do presente trabalho, o principal problema está relacionado com a redução habitual do limite de resistência e da tenacidade com a execução do TTPS [21,22,54,55,57,66,69].

A este respeito, o desenvolvimento de procedimentos de soldagem seguros para aços de elevada resistência utilizados em componentes das linhas de ancoragem apresentam uma enorme relevância, devido à necessidade de fabricar e reparar muitos componentes industriais que seriam descartados pela existência de defeitos de fabricação.

Como já comentado, embora existam evidências de consumíveis comerciais adequados para esta tarefa $[8,19,21,22,27,28,31,41]$, é importante ressaltar que estes nem sempre estão disponíveis no mercado brasileiro quando um reparo urgente tem que ser realizado. Além do custo muito alto do próprio material, o tempo de entrega para importar o consumível é uma questão crítica. Assim, o desenvolvimento de tecnologia local para produzir o material é da maior importância, tal como já se observou em um trabalho de soldagem de manilhas fabricadas em aços fundidos de alta resistência de grau R4 [13], utilizando um consumível com composição desenvolvida no presente programa de pesquisa.

No presente programa, a composição química com base em maiores teores de $\mathrm{Mn}$-Ni e baixos teores de $\mathrm{Cr}$, embora apresentando tenacidade ao impacto inferior no estado de como soldado, foi capaz de garantir propriedades adequadas após a execução do TTPS. É fundamental reforçar esta questão, porque a literatura sobre a influência da TTPS nas características de metais de solda de alta resistência ainda é limitada, dado que o interesse principal dos fornecedores de consumíveis é a aprovação no estado como soldado, já os requisitos após TTPS não são previstos. Isto toma importância maior quando se nota que um mesmo consumível pode apresentar comportamentos totalmente diferentes antes e após TTPS, ou seja, a aprovação no estado como soldado não é garantia de seu desempenho em projetos submetidos aos tratamentos térmicos obrigatórios para alívio das tensões residuais das estruturas. 


\section{CONCLUSÕES}

Com base nas condições experimentais aplicadas no presente trabalho, as principais conclusões são:

a) O metal de solda com composição química contendo 2,0Mn-3,0Ni e baixo teor de $\mathrm{Cr}$ é capaz de manter altos níveis de resistência mecânica e resistência ao impacto após o TTPS;

b) O TTPS promoveu uma ligeira redução na resistência mecânica e uma melhora na tenacidade ao impacto;

c) $\mathrm{O}$ aumento do tempo de tratamento promoveu mudanças significativas nas propriedades mecânicas;

d) O metal de solda de aço de alta resistência apresentando uma composição química baseada em 2,0Mn3,0Ni atingiu os requisitos da norma IACS W22 para aços grau R3 e R4, dependendo do TTPS aplicado e;

e) O desenvolvimento de um consumível nacional adequado para a soldagem de aços de elevada resistência utilizados em componentes de ancoragem pode ser um fator importante para a redução de custos, já que irá contribui para evitar o sucateamento prematuro de equipamentos com defeitos de fabricação.

\section{AGRADECIMENTOS}

Os autores agradecem ao CEFET / RJ, PUC-RIO, COPPE-UFRJ, CNPq e CAPES pelo apoio na execução do presente trabalho.

\section{BIBLIOGRAFIA}

[1] CHENG, X., CHEN, H., LIU, W., et al., Influence of mooring chain steel strength on stress corrosion cracking, Applied Mechanics and Materials, v. 404, pp.32-39, 2013.

[2] OZMUTLU, S., "The use of drag embedment anchors in offshore applications", Proc. Int. Conf. on 'Offshore Renewable Energy', Porto, Pt, May 2012.

[3] CARNEIRO, M.A., PEREIRA, M.V.S., DARWISH, F.A., et al., "Fatigue crack propagation in structural steel after flash welding", Welding International, v.23, n.3, pp.151-158, 2009.

[4] COSTA, L.C.S., CASTRO, G.A.V., GONÇALVES, R.C.F., et al., "Polyester mooring systems Petrobras Experience", Proc. of the 'Deep Offshore Technology Conference, Rio de Janeiro, Brazil, September 2001.

[5] KIVTRUD, A., "Lessons learned from the norwegian mooring lines failures 2010-2013", In: Proc. of the $33^{\text {rd }}$ Int. Conf. on 'Ocean, Offshore and Arctic Engineering, San Francisco, USA, June 2014.

[6] KITAGAWA, Y., KAWASAKI, H., "Recent Development of High-strength and tough welding consumables for offshore structures", Kobelco Tech. Rev., v. 32, pp. 1-8, 2013.

[7] IACS W22 International Association of Classification Societies. Specification W22, Offshore Mooring Chain. London, IACS, 2016.

[8] SANTOS FILHO, O.R., DINIZ, J.L.C., COELHO, M.J.S., et al., "Avaliação de juntas soldadas de um aço fundido de alta resistência para a indústria offshore", Soldagem \& Inspeção, v.23, n.2, pp.205-224, 2018.

[9] WANG, Z., QI, J., LIU, Y., "Effect of silicon content on the hardenability and mechanical properties of link-chain steel", Journal of Materials Engineering and Performance, v.28, pp.1678-1684, 2019.

[10] IDAPALAPATI, S., AKISANYA, A.R., LOH, K.M., et al., Failure analysis of a failed anchor chain link, Engineering Failure Analysis 2017, DOI:10.1016/j.engfailanal.2018.03.007

[11] LEAL, S.N.C.C., "Corrosão em elos de amarras offshore: um estudo de caso", Dissertação M.Sc., Programa de Pós-Graduação em Engenharia Metalúrgica do Departamento de Ciências dos Materiais e Metalurgia da PUC-Rio, outubro, 155p, 2003.

[12] JORGE, J.C.F., SOUZA, L.F.G., SANTOS FILHO, O.R., et al., "Relação tenacidade/microestrutura da ZTA de aço fundido ASTM A 148 Gr. 80-50 para acessórios de ancoragem de plataformas de petróleo", Soldagem \& Inspeção, v.9, n.4, pp.192-197, 2004.

[13] SUMAM, J.A., JORGE.J.C.F., SOUZA, L.F.G., et al., "Efeito de tratamentos térmicos pós-soldagem nas propriedades de aço fundido de elevada resistência para sistemas de ancoragem de plataformas marítimas", Soldagem \& Inspeção, v.9, v.4, pp.205-212, 2004.

[14] SALVADOR, L.S., "Efeito da composição química e tratamento térmico pós-soldagem nas propriedades de metal de solda de aços de alta resistência”, Dissertação M.T., CEFET-RJ, 1997. 
[15] COELHO, W.P., NOGUEIRA, Y.RG., Soldagem do aço 8630 modificado, TCC, dezembro, Curso de Pós-Graduação em Engenharia de Soldagem , UFMG, 2015, 93p.

[16] INTERNATIONAL ASSOCIATION OF CLASSIFICATION SOCIETIES. W8, Hull and machinery steel castings. London, IACS, 2004.

[17] INTERNATIONAL ASSOCIATION OF CLASSIFICATION SOCIETIES. W28, Welding procedure qualification tests of steels for hull construction and marine structures. London, IACS, 2012.

[18] AMERICAN BUREAU OF SHIPPING. Rules for Building and Classing Steel Vessel Rules Part 2, Rules for Materials and Welding. Houston, ABS, pp. 40-44. Chapter 1: Materials for Hull Construction, Section 5 Hull Steel Casting, 2013.

[19] OLIVEIRA, E.M., JORGE. J.C.F., SOUZA, L.F.G., "Efeito de tratamento térmico pós-soldagem nas propriedades mecânicas de juntas soldadas de aço fundido de alta resistência mecânica", In: Anais do $69^{\circ}$ Congresso Internacional da ABM, Jul 21-25; São Paulo, Brasil. São Paulo, Associação Brasileira de Metalurgia e Materiais, pp.1-12, 2014.

[20] AMERICAN WELDING SOCIETY. Specification for low alloy steel electrodes for shielded metal arc welding - AWS 5.5. Miami, AWS, 1996.

[21] GOMES, A.J.M., JORGE. J.C.F., SOUZA, L.F.G., et al., "Influence of chemical composition and post welding heat treatment on the microstructure and mechanical properties of high strength steel weld metals", Materials Science Forum, v.759, pp.21-32, 2013.

[22] JORGE, J.C.F., FARAGASSO, S.M., SOUZA, L.F.G., et al., "Effect of post welding heat treatment on the mechanical and microstructural properties of extra high-strength steel weld metals for application on mooring equipment", Welding International, v. 29, pp.521-529, 2015.

[23] JORGE, J.C.F., et al., "Influence of welding procedure and PWHT on HSLA steel weld metals", Journal of Materials Research and Technology, v.8, n.1, pp.561-571, 2019.

[24] FARNEZE, H.N., JORGE. J.C.F., SOUZA, L.F.G., BOTT, I.S., "Comparative study of high-strength steel weld metals obtained by the SMAW and FCAW processes for offshore applications and mooring chains", Welding International, v.24, pp.903-910, 2010.

[25] LINS JUNIOR, A.S., COSTA, H.R.M., SOUZA, L.F.G., et al., "Propriedades mecânicas e microestruturais de juntas soldadas do aço HY-80 pelos processos eletrodo revestido e GMAW", Soldagem \& Inspeção, v.19, n.3, pp.200-211, 2014

[26] LORD, M., JENNINGS, G. "Effect of interpass temperature on properties of high-strength weld metals", Svetsaren, v.54, n.1-2, pp.53-58, 1999.

[27] KARLSSON, L., KEEHAN, E., ANDREN, H.O., et al., "Development of high strength steel weld metals -potential of novel high-Ni compositions", Proc. Of the Eourojoin, v. 5, pp.1-8, 2004.

[28] KEEHAN, E., ZACHRISSON, J., KARLSSON, L., "Influence of cooling rate on microstructure and properties of high strength steel weld metal", Science and Technology of Welding and Joining, v.15, n.3, pp.233-238, 2010.

[29] JORGE, J.C. F., SOUZA, L.F.G, SANTOS FILHO, O.R., et al., "Influência da composição química e tratamento térmico pós-soldagem nas propriedades mecânicas e microestruturais de metais de solda de alta resistência", In: Anais do XXXIII CONSOLDA, pp.1-10, 2007.

[30] GOMES, A.J.C., QUEIROZ, S.S., JORGE, J.C.F., et al., "Propriedades mecânicas e microestrutura de metais de solda de alta resistência obtidos pelos processos GMAW e SMMAW", In: Anais do $72^{\circ}$ Congresso Anual da ABM, v.72, n.1, pp.1177- 1186, 2017.

[31] GOMES, A.J.C., "Estudo da relação tenacidade x microestrutura de metais de solda de aços de alta resistência submetidos a tratamentos térmicos", Dissertação M.Sc., CEFET-RJ, 2018.

[32] MOURA, D.S., "Porpriedades mecânicas e microestuturais de metl de solda de alta resistência submetido a tratamentos térmicos", Dissertação M.Sc., CEFET-RJ, abril, 2016.

[33] ISO 15792-1. Part 1: Test methods for all-weld metal test specimens in steel, nickel and nickel alloys, First Edition, 2000.

[34] ASTM International. Standard test methods and definitions for mechanical testing of steel products, ASTM A-370-05. West Conshohocken, USA, ASTM, 2005.

[35] ASTM International. Standard test methods for tension testing of metallic materials, ASTM E8. West Conshohocken, USA, ASTM, 2005. 
[36] ASTM International. Standard test methods for notched bar impact testing of metallic materials, ASTM E23. West Conshohocken, USA, ASTM, 2005.

[37] DIAS-FUENTES, M., IZA-MENDIA, A., GUTIERREZ, I., "Analysis of different acicular ferrite microstructures in low-carbon steels by electron backscattered diffraction. Study of their toughness behavior", Metallurgical and Materials Transactions A, v.34A, pp.2505-2516, 2003.

[38] WANG, Y., KANNAN, R., ZHANG, L., LI, L., "Microstructural analysis of the as-welded heat-affected zone of a grade 91 steel heavy section weldment", Welding Journal, v. 96, n.6, pp.203s-219s, 2017.

[39] SURIAN, E.S., RISSONE, N.M.R., SVOBODA, H.G., et al., "FCAW and SAW high-strength ferritic deposits: the challenge is tensile properties", Welding Jorurnal, v.89, n.3, pp.54s-64s, 2010.

[40] WANG, H.H., LI, G.Q., WAN, X.L., et al., "Microstructural characteristics and impact toughness in YS690MPa steel weld metal for offshore structures", Science and Technology of Welding and Joining, v.22, n. 2, pp. 133-142, 2017.

[41] KEEHAN, E., KARLSOON, L., ANDREN, H.O., "Influence of carbon, manganese and nickel on microstructure and properties of strong steel weld metals, Part 1-effect of nickel content", Science and Technology of Welding and Joining, v.11, pp.1-8, 2006.

[42] LEE, K.H., PARK, S.G., KIM, M.C., et al., "Cleavage fracture toughness of tempered martensitic NiCrMo low alloy steel with different martensite fraction", Materials Science and Engineering A, v.534, pp.75- 82, 2012.

[43] JABERI, F.S., KOKABI, A.H., "Influence of nickel and manganese on microstructure and mechanical properties of shielded metal arc-welded API-X80 steel", Journal of Materials Engineering and Performance, v.21, pp.1447-1454, 2012.

[44] MAO, G., CAO, R., CAYRON, C., et al., "Microstructural evolution and mechanical property development with nickel addition in low-carbon weld butt joints", Journal of Materials Processing Technology, v.262, pp.638-649, 2018.

[45] EVANS, G.M., Predictive Charpy-V toughness of the manganese-nickel weld metal combination, Doc.II-C-546-18 Genoa, Italy, March, 2018.

[46] ZHANG, Z., FARRAR, R.A., "Influence of Mn and Ni on the Microstructure and Toughness of C-MnNi Weld Metals", Welding Journal, v.76, n.5, pp.183-195, 1997.

[47] KANG, B.Y., KIM, H.J., "Effect of Mn and Ni on the Variation of the Microstructure and Mechanical Properties of Low-Carbon Weld Metal", ISIJ InternationaL, v.40, n.12, pp.1237-1245, 2000.

[48] HOLLY, S., HASLBERGER, P., ZUGNER, D., et al., "Development of high-strength welding consumables using calculations and microstructural characterization", Welding in the World, v. 62, n.3, pp.451-458, 2018.

[49] KARLSSON, L., SVENSSON, L.E., HURTIG, K., "Influence of dilution on properties of high strength steel weld metals", Biuletyn Instytutu Spawalnictwa, v.5, pp.62-70, 2014.

[50] GIANETTO, J.A., SMITH, N.K., MCGRATH, J.T., et al., "Effect of composition and energy input on structure and properties of high-strength weld metals", Welding Journal, v.72, n.11, pp.407s-419s, 1992.

[51] ZHANG, T., LI, Z., MA, S., et al., "High strength steel (600-900 MPa) deposited metals: microstructure and mechanical properties", Science and Technology of Welding and Joining, v.21, n.3, pp.186-193, 2016.

[52] SCHNITZER, R., ZUGNER, D., HASLBERGER, P., et al., "Influence of alloying elements on the mechanical properties of high-strength weld metal", Science and Technology of Welding and Joining, v.22, pp.536-543, 2017.

[53] BEIDOKHTI, B., POURIAMANESH, R., "Effect of filler metal on mechanical properties of HSLA welds", Welding Journal, v.94, n.10, pp.334s-341s, 2015.

[54] SURIAN, E.S., TROTTI, J., CASSANELLI, A., et al., "Influence of chromium on the mechanical properties and microstructure of weld metal from a high strength SMA electrode", Welding Journal, v. 74, n.3, p.45s-53s, 1994.

[55] SURIAN, E.S., TROTTI, J., HERRERA, R., et al., "Influence of carbon on mechanical properties and microstructure of weld metal from a high-strength SMA electrode", Welding Journal, v.70, n.10, pp.133s140s, 1991.

[56] KEEHAN, E., KARLSSON, L., ANDRÉN, H.O., "Influence of carbon, manganese and nickel on microstructure and properties of strong steel weld metals, Part 1-effect of nickel content", Science and Tech- 
nology of Welding and Joining, v.11, pp.1-8, 2006.

[57] SURIAN, E.S., RISSONI, M.R., de VEDIA, L.A., "Influence of Molybdenum on Ferritic High-Strength SMAW All-Weld-Metal Properties", Welding Journal, v.85, n.4, pp.53s-62s, 2005.

[58] LEE, H., "Effect of Cr content on microstructure and mechanical properties of low carbon steel welds", International Journal of Electrochemical Science, v.10, pp.8028-8040, 2015.

[59] KIM, S., LEE, E., CHOI, Y., et al., "A study on the material characteristics and the welding properties of the HSA800 steel", International Journal of Steel Structures, v.17, n.2, pp.821-831, 2017.

[60] LAN, L., KONG, X., QIU, C., "Characterization of coarse bainite transformation in low carbon steel during simulated welding thermal cycles", Materials Characterization, v.105, pp. 95-103, 2015.

[61] PARK, S., KIM, M., LEE, B., et al., "Correlation of the thermodynamic calculation and the experimental observation of NiMoCr low alloy steel changing $\mathrm{Ni}$, Mo, and $\mathrm{Cr}$ contents", Journal of Nuclear Materials, v. 407, pp.126-135, 2010.

[62] AVAZKONANDEH-GHARAVOL, M.H., HADDAD-SABZEVAR, E.M., HAERIAN, E.A., Effect of chromium content on the microstructure and mechanical properties of multipass MMA, low alloy steel weld metal, Journal of Materials Science, v.44, pp.186-197, 2009.

[63] JORGE, J.C.F., SOUZA, L.F.G., REBELLO, J.M.A., et al., "Effect of chromium on the mechanical properties of Mn-Mo steel welds containing titanium, IIW DOC. II-1398-00", In: Conference IIW Annual Assembly, At: Florence, Italy, June, 2000, Doc.II-1398-00.

[64] WANG, Q., LI, C., PENG, H., CHEN, J., et al., "Effect of tempering temperature on the microstructure and properties of Fe-2Cr-Mo-0.12C pressure vessel steel", Journal of Materials Engineering and Performance, v.27, pp.1485-1493, 2018.

[65] PENG, Y., ZHANG, Y., ZHAO, L., et al., "Effect of heat input and heat treatment on microstructure and mechanical properties of welded joint of TMCP890 steel", Welding in the World, v.62, n.5, pp.961-971, 2018.

[66] BAUNÉ, E., CHOVET, C., LEDUEY, B., et al., Consumables for welding of (very) high strength steels mechanical properties of weldments in as-welded and stress-relieved applications, IIW Doc. II-1696-06, 2006.

[67] RAMIREZ, J.E., "Characterization of high strength steel weld metals, chemical composition, microstructure and nonmetallic inclusions", Welding Journal, v.87, n.5, pp.65s-75s, 2008.

[68] ZHANG, T., LI, Z., YOUNG, F., et al., "Global progress on welding consumables for HSLA steel”, ISIJ Int., n.54, pp. 1472-1484, 2014.

[69] JORGE, J.C.F., SOUZA, L.F.G., SANTOS FILHO, O. R., et al., "Estudo de metais de solda de extra alta resistência para aplicação em componentes de ancoragem de plataformas de petróleo", Parte I: propriedades mecânicas, In: Anais do $37^{\circ}$ Congresso Brasileiro de Soldagem, Natal, Brasil, Outubro, pp. 1-10, 2011.

\section{ORCID}

Antonio José de Carvalho Gomes

Jorge Carlos Ferreira Jorge

Luís Felipe Guimarães de Souza

Ivani de Souza Bott

Leonardo Sales Araújo

Matheus Campolina Mendes http://orcid.org/0000-0002-7932-4651

http://orcid.org/0000-0001-8312-0312

http://orcid.org/ 0000-0002-6669-8451

http://orcid.org/0000-0002-7120-978X

http://orcid.org/0000-0001-5564-0695

http://orcid.org/0000-0001-7755-2784 